Published in final edited form as:

Adv Healthc Mater. 2016 February 04; 5(3): 373-381. doi:10.1002/adhm.201500451.

\title{
Ultrathin Injectable Sensors of Temperature, Thermal Conductivity, and Heat Capacity for Cardiac Ablation Monitoring
}

\author{
Dr. Ahyeon Koh, \\ Department of Materials Science and Engineering, Frederick Seitz Materials Research \\ Laboratory, University of Illinois at Urbana-Champaign, Urbana, IL 61801, USA \\ Dr. Sarah R. Gutbrod, \\ Department of Biomedical Engineering, Washington University in Saint Louis, Saint Louis, MO \\ 63130, USA

\section{Dr. Jason D. Meyers,} \\ Department of Biomedical Engineering, Washington University in Saint Louis, Saint Louis, MO \\ 63130, USA
}

\section{Prof. Chaofeng Lu,}

Department of Civil Engineering and Soft Matter Research Center, Zhejiang University, Hangzhou 310058, China

Department of Mechanical Engineering and Civil and Environmental Engineering, Northwestern University, Evanston, IL 60208, USA

\section{Dr. Richard Chad Webb,}

Department of Materials Science and Engineering, Frederick Seitz Materials Research

Laboratory, University of Illinois at Urbana-Champaign, Urbana, IL 61801, USA

\section{Dr. Gunchul Shin,}

Department of Materials Science and Engineering, Frederick Seitz Materials Research

Laboratory, University of Illinois at Urbana-Champaign, Urbana, IL 61801, USA

\section{Yuhang Li,}

Institute of Solid Mechanics, Beihang University, Beijing 100191, China

\section{Dr. Seung-Kyun Kang,}

Department of Materials Science and Engineering, Frederick Seitz Materials Research Laboratory, University of Illinois at Urbana-Champaign, Urbana, IL 61801, USA

\section{Prof. Yonggang Huang,}

Department of Mechanical Engineering and Civil and Environmental Engineering, Northwestern University, Evanston, IL 60208, USA

Prof. Igor R. Efimov, and

\footnotetext{
Correspondence to: Igor R. Efimov; John A. Rogers.

A.K., S.R.G., and J.D.M. contributed equally to this work.

Supporting Information

Supporting Information is available from the Wiley Online Library or from the author.
} 
Department of Biomedical Engineering, Washington University in Saint Louis, Saint Louis, MO 63130, USA

Department of Biomedical Engineering, George Washington University, Washington, DC 20052, USA

Prof. John A. Rogers

Department of Materials Science and Engineering, Frederick Seitz Materials Research Laboratory, University of Illinois at Urbana-Champaign, Urbana, IL 61801, USA

\section{Abstract}

Knowledge of the distributions of temperature in cardiac tissue during and after ablation is important in advancing a basic understanding of this process, and for improving its efficacy in treating arrhythmias. Technologies that enable real-time temperature detection and thermal characterization in the transmural direction can help to predict the depths and sizes of lesion that form. Herein, materials and designs for an injectable device platform that supports precision sensors of temperature and thermal transport properties distributed along the length of an ultrathin and flexible needle-type polymer substrate are introduced. The resulting system can insert into the myocardial tissue, in a minimally invasive manner, to monitor both radiofrequency ablation and cryoablation, in a manner that has no measurable effects on the natural mechanical motions of the heart. The measurement results exhibit excellent agreement with thermal simulations, thereby providing improved insights into lesion transmurality.

\section{Introduction}

Over the past several decades, catheter ablation technology has emerged as a dominant mode for eliminating arrhythmias, and is now widely considered as a first line of treatment. Depending on the type of arrhythmia, observed success rates can be as low as $65 \%$ with a single procedure. ${ }^{[1]}$ The goal of catheter ablation is to create controlled lines of damage in a diseased heart to insulate healthy tissue from abnormal electrical activity. Any healthy tissue remaining along the width or depth of a lesion results in a failure. A major clinical challenge stems from the lack of physiological feedback during lesion formation, due to an inability to directly measure tissue temperature or track lesion depth during formation. ${ }^{[2]}$ Catheter technology has advanced recently through the introduction of force-sensing elements, ${ }^{[3]}$ which provide data relevant to physical contact between a catheter and the myocardium. Real-time direct monitoring of tissue characteristics and indicators of lesion formation during ablation remains, however, an unmet need.

Recent research focuses on various classes of temperature sensors for applications in ablation monitoring, where tissue compatible mechanics and materials offer precision measurements and high spatiotemporal resolution. These devices effectively measure temperature on the endocardial or epicardial surfaces, and exist at the system level either as 3D multifunctional integumentary membranes, ${ }^{[4]}$ inflatable balloon catheters, ${ }^{[5]}$ or ultralow modulus stretchable epicardial webs. ${ }^{[6]}$ In these devices, thin metal thermistors rest on flexible or stretchable polymer substrates, to allow conformal contact on the curved surfaces of the tissues, without mechanical constraint. A key limitation, however, is that the 
measurements reveal thermal distributions on surfaces, with only indirect information on the lesion depth or size. During ablation, the tissue response within the myocardial wall is critical to successful outcomes.

Herein, we introduce classes of injectable thermal sensors that are compatible with comparatively stiff, and highly time dynamic cardiac tissue, for the purpose of real-time monitoring of temperature, thermal conductivity $(k)$, and volumetric heat capacity $\left(\rho C_{\mathrm{p}}\right)$ at several depths during lesion formation using both radiofrequency (RF) ablation and cryoablation (Figure 1J). The formats build on recent concepts in injectable and flexible electronics and optoelectronics, where thin polymer needles serve as vehicles for delivering semiconductor devices into the depths of the brain for experiments in optogenetics. ${ }^{[7]}$ Removable guide needles allow the use of ultrathin substrates, which, due to their low bending stiffnesses, would not otherwise effectively penetrate the cardiac tissue. Such ultrathin geometries minimize tissue damage during insertion and do not impede cardiac contraction. Low bending stiffnesses and thermal masses ensure negligible effects on the thermal or mechanical properties of the tissue. The real-time changes in temperature along with pre- and postablation measurements of $k$ and $\rho C_{\mathrm{p}}$ of tissue recorded from inside the myocardial wall correlate strongly to lesion transmurality and simulation models of the thermal physics.

\section{Results and Discussion}

\subsection{Ultrathin Injectable Sensors of Temperature and Thermal Transport}

The sensors rely on serpentine lines of $\mathrm{Au}$ ( $150 \mathrm{~nm}$ thick) photolithographically defined on thin substrates of polyethylene terephthalate (PET; $6 \mu \mathrm{m}$ thick) patterned into needlelike shapes. ${ }^{[7,8]}$ The Experimental Section provides details. A schematic illustration of the layout and a corresponding optical image appear in Figure 1. The needles support three such multimodal sensors, each of which occupies an area of $0.4 \mathrm{~mm} \times 0.4 \mathrm{~mm}$, spaced $1 \mathrm{~mm}$ apart. A four-wire contact configuration reduces parasitic errors caused by changes in resistances of the interconnections. A layer of a photocurable epoxy (SU-8, $5 \mu \mathrm{m}$ thick) serves as encapsulation. The electrical resistance of each sensor determines the temperature, via the temperature coefficient of resistance (TCR) of the metal. Optical and X-ray computed tomography (Micro-XCT; Xradia, CA) images appear in Figure 1D,F, respectively. Thermal conductivity $(k)$ and volumetric heat capacity $\left(\rho C_{\mathrm{p}}\right)$ can be determined by using the same sensors to induce precise levels of Joule heating and to evaluate the time dynamics of the resulting changes in temperature. ${ }^{[9]}$

In vitro testing establishes the thermal responses and mechanical properties of the sensors. The change in resistance $(\Delta R)$ is directly proportional to the change in temperature, within physiological ranges, via the TCR as determined by calibration against a commercial thermocouple. The experimentally determined TCR is $0.26 \pm 0.01 \%{ }^{\circ} \mathrm{C}^{-1}$, where the error bar corresponds to device-to-device variations. The precision, as defined by the repeatability of measurements with a given sensor, corresponds to a temperature change of $\approx 0.1{ }^{\circ} \mathrm{C}$ at a sampling rate of $4 \mathrm{~Hz}$. The calibration curves, shown in Figure $1 \mathrm{G}$, are highly linear $\left(R^{2}=\right.$ 0.99 ) within the biologically relevant range, with a average slope of $0.51 \Omega{ }^{\circ} \mathrm{C}^{-1}$ (standard error of mean $=0.02 ; n=28$ ). 
The same sensor enables evaluation of the thermal conductivity and diffusivity. ${ }^{[8]}$ Here, flowing current through the sensor induces precise and minute increases in temperature by Joule heating. The time dynamics of the associated changes in temperature before, during, and after this heating can be quantitatively connected to key thermal properties via analysis algorithms described elsewhere. ${ }^{[8]}$ The thermal conductivity $(k)$ and volumetric heat capacity $\left(\rho C_{\mathrm{p}}\right)$ determined in vitro using this method with chicken breast at $37^{\circ} \mathrm{C}$ are 0.53 $\mathrm{W} \mathrm{m}{ }^{-1}{ }^{\circ} \mathrm{C}^{-1}$ and $3.55 \mathrm{~J} \mathrm{~cm}^{-3}{ }^{\circ} \mathrm{C}^{-1}$, respectively. The percent standard errors of the mean are 1.6 and $3.7 \%$ for $k$ and $\rho C_{\mathrm{p}}$, respectively. In this way, the needle-type flexible devices reported here offer quantitative in situ analytical determinations not only of temperature but also of important thermal transport properties.

The devices have total thicknesses of $\approx 11 \mu \mathrm{m}$, and bending stiffnesses of $\approx 21.0 \mathrm{~N} \mathrm{~m}^{-1}$ for frequencies between 0.1 and $10 \mathrm{~Hz}$, corresponding to typical cardiac contractions and respiratory rates (Figure $1 \mathrm{H}$ ). The calculated thermal mass of the part of the device that implants into the tissue $(\approx 5 \mathrm{~mm})$ is $45 \mu \mathrm{J}^{\circ} \mathrm{C}^{-1}$ corresponding to an equivalent thermal mass of $\approx 10 \mathrm{~nL}$ of water. This low thermal mass allows rapid response to changes in temperature during ablation, without significant influences on the natural temperature variations. The experimentally determined response time $\left(t_{90}\right)$ is $\approx 10 \mathrm{~ms}$ (Figure S1, Supporting Information), thereby providing a temporal resolution needed for characterizing changes in temperature associated with the ablation process. In this study, real-time measurements of temperature provide data of direct relevance to the cardiac ablation procedure. Additional capabilities in monitoring $k$ and $\rho C_{\mathrm{p}}$ before and after this process, with the same sensors, have potential relevance in detecting morphological or chemical changes in the tissue, of value not only in ablation but also in other surgical contexts. Tissue transmurality, confirmed by postablation histological staining with analytical thermal modeling, represents information for predicting the ablation efficacy in ways that could lead to real-time feedback for a physician.

\subsection{Transmural Spatiotemporal Temperature Determination during Cardiac Ablation}

Measurements involve insertion of the devices into the left ventricular (LV) wall of a perfused rabbit heart from the exposed endocardial surface. The extreme mechanical flexibility of the device necessitates the use of a tungsten guide needle ( $50 \mu \mathrm{m}$ thick) to enable penetration into the tissue. After implantation, dissolution of a cellulose adhesive layer allows removal of this guide needle, thus leaving only the temperature sensors embedded within the myocardial tissue. An example of an ex vivo heart preparation with the guide needle removed is shown in Figure 1I. Details of the sensor insertion procedure are illustrated in Figure S2 (Supporting Information). Insertion locates the device at a depth of $\approx 3.5 \mathrm{~mm}$ within the transmural wall, with the proximal sensor (i.e., sensor 3) positioned just below the endocardial tissue surface and the distal temperature sensor (i.e., sensor 1) at a depth of $2.2 \mathrm{~mm}$. The platform provides real-time temperature data at different depths during ablation procedures (Figure 2). The extremely high degree of mechanical flexibility ensures biomechanical compatibility, minimizing tissue damage during natural cardiac contractions (Video S1, Supporting Information). 
In current procedures, the only thermal feedback available to the physician is a thermocouple or thermistor in the shaft of the ablation catheter. Because such sensors can be millimeters away from the tissue surface, they cannot capture critical information on the temperature distributions along the transmural wall below the surface of the tissue. The sensors presented here embed into the wall, to enable measurements that reveal changes in temperature during both cryoablation and RF ablation. Demonstrations with commercial ablation catheters confirm that the sensors offer required performance over the full range of clinically relevant temperatures. The data yield chronothermographs at three locations in the transverse direction, thereby characterizing the distribution of thermal energy across the wall during lesion formation. This thermal analysis yields real-time, localized changes in temperature in the myocardial tissue, inaccessible to techniques such as IR thermography (Video S2, Supporting Information). The stark differences in the temperature profiles measured by the catheters and those determined by implanted sensors for a representative 10 W transmural lesion appear in Figure S4 (Supporting Information).

For both cryo- and low power ( 5 and $10 \mathrm{~W}$ ) RF ablation, the greatest and fastest changes in temperature occur at the position of the sensor located proximal to the ablation source. Positions into the depth of the myocardial tissue show progressively smaller and slower changes, as shown in Figure 2A,B. High power (i.e., >20 W) RF ablation yields less distinctive thermal distributions (Figure S3, Supporting Information). The thermographs of both ablation methods show clear mechanistic phases of lesion formation. The temperature profiles from cryoablation reveal definitive freezing and thawing periods across all three sensors, while the RF ablation temperature profiles reveal clear inflection points.

Mechanistically, RF ablation creates the desired tissue damage in two ways. First, the induced electrical currents directly heat the tissue through resistive heating. The degree of heating decreases with distance from the current source, in a manner that follows the current distributions. Second, passive conductive heat transfer through the tissue causes an overall spreading of the thermal distribution. ${ }^{[10]}$ The inflection points in the RF temperature profiles likely represent transitions from resistive to conductive heating (Figures 2A). Differences captured by these implanted sensors could improve our fundamental understanding of tissue response to various stages of thermal intervention and aid in the development of novel treatment strategies.

Simultaneous use of multiple sensors injected at different locations allows determination of the lateral distributions in temperature during RF ablation (10 W, Figure 2C). These data involve two additional sensors at sites 2 and $5 \mathrm{~mm}$ away from the ablation source. As expected, sensors located relatively far from the ablation source show minor, and gradual changes in temperature, confirming the signals are localized. The collective results provide insights into the full 3D temperature profiles.

The values of $k$ and $\rho C_{\mathrm{p}}$ evaluated in cardiac tissue using this same measurement platform are $0.43 \pm 0.02 \mathrm{~W} \mathrm{~m}^{-1}{ }^{\circ} \mathrm{C}^{-1}$ and $2.8 \pm 0.1 \mathrm{~J} \mathrm{~cm}^{-3}{ }^{\circ} \mathrm{C}^{-1}$, respectively. These parameters, which are important in analytical modeling of the thermal distribution, ${ }^{[11]}$ are approximately the same before and after both hypo- and hyperthermic cardiac ablation (Figure 2D). 
Table 1 summarizes the details of temperature changes during a nontransmural RF ablation (i.e., $5 \mathrm{~W}$ power). The temperature sensor proximal to the ablation source (i.e., sensor 3 ) indicates larger temperature changes and rates of temperature change compared to those at depths corresponding to the tip of the needle (i.e., sensor 1). The maximum temperatures measured by the temperature sensors at the distal tip (i.e., sensor 1 ) and the middle (i.e., sensor 2 ) of the needle are $\approx 12 \%$ and $6 \%$ less, respectively, than that measured proximal to the ablation source (i.e., sensor 3). The initial slope of the temperature change (estimated as linear) exhibits a similar trend, i.e., increasing with decreasing distance to the source and the tissue surface. The corresponding slopes are $0.21,0.41$, and $0.62{ }^{\circ} \mathrm{C} \mathrm{s}^{-1}$ for sensors 1,2 , and 3 , respectively. As expected, the temperature changes, including the maximum temperatures, increase with ablation power, consistent with measurements of temperature distributions on the surface.

Experimental RF lesions $(n=20)$ and an analytical model allow correlation of temperature profiles with lesion depths. The calculated temperature distributions during RF ablation appear in Figure 3C-E. The model is similar to those described previously, ${ }^{[6]}$ with details in the Supporting Information. Owing to the forced convection associated with fluid flow in the bath surrounding the heart (a large and random value of convection coefficient $h_{\mathrm{c}}$ ), the results from the sensor 3 (implanted closest to the tissue surface) were excluded for computational fitting (Video S2, Supporting Information and Figure 3F). These flows induced complex patterns of convection at the endocardial surface, such that surface measurements of temperature do not offer reliable correlations to numerical simulations of the through-depth temperature distribution. Experimentally determined changes in temperature from the fully implanted sensors (i.e., located at the middle and distal tip, sensor 2 and 1, respectively) provide excellent agreement with analytical calculations and finite element analysis (FEA) for ablation at RF powers from 5 to $20 \mathrm{~W}$, as shown in Figure 3C-E.

\subsection{Assessment of Transmurality of Cardiac Ablation Lesions}

A range of ablation powers combined with intrinsic variations in ventricular geometry result in a distribution of lesion transmurality, as shown in Figure 4. The lesions can be grouped into fully transmural (clinical success) $(n=11)$ and nontransmural (clinical failure) $(n=9)$ cases. A representative example of a nontransmural and a transmural lesion appear in Figure 4A,B with the lesion depths identified. The shaded region highlights the lesion as defined by a custom threshold program described in the Experimental Section. The corresponding temperature profiles for all lesions can be assessed to extract parameters that may aid in the real-time identification of successful lesion attempts. Figure 5E shows the temperature change recorded during $\mathrm{RF}$ ablation at $5 \mathrm{~W}$, along with definitions of the parameters used to characterize the shape of the thermograph profile. Details appear in the Experimental Section. Four key para meters include: (1) maximum change in temperature $\left(\Delta T_{\mathrm{M}}\right),(2)$ time spent in the initial linear phase of heating (phase 1) $\left(t_{\mathrm{P} 1}\right),(3)$ ratio of the change in temperature at the inflection point to the maximum change in temperature $\left(R_{\mathrm{TP} 1}\right)$, and (4) ratio of the time of the inflection point to the time of the maximum temperature change $\left(R_{\mathrm{tP} 1}\right)$. The first inflection point and the maximum change in temperature appear as orange and green, respectively (Figure 5E). Each parameter can be statistically compared by location within the myocardial wall and across the transmurality groups. The two parameters 
with statistically different results across the transmural groups are the $\Delta T_{\mathrm{M}}(p=0.0039)$ and the $R_{\mathrm{tP} 1}(p=0.0328)$. Across all sensors, transmural lesions demonstrate a significantly higher $\Delta T_{\mathrm{M}}$ and a higher $R_{\mathrm{tP} 1}$ (i.e., more time spent in resistive heating) compared to those of nontransmural lesions. Consistent with the modeling data, the middle sensor (i.e., sensor 2) revealed the greatest differences between transmural and nontransmural lesions for all parameters except maximum temperature (Figure 5F) and may therefore be the best sensor for lesion characterization.

Receiver operator curves (ROCs) and the area under these curves (AUCs) for all four parameters are presented in Figure 6 and Table 2, respectively. These curves demonstrate that the most accurate determination of transmurality corresponds to the $t_{\mathrm{P} 1}$ at the middle sensor and the $\Delta T_{\mathrm{M}}$ at the most proximal sensor. When evaluating these two parameters for clinical use in helping to guide ablation in real-time, it is important to note that while $\Delta T_{\mathrm{M}}$ can only be examined post hoc after lesion creation, resistive heating $\left(R_{\mathrm{tP} 1}\right)$ can be observed during lesion formation. Therefore, these observations suggest that transmurality of lesion can be most easily and reliably determined using $R_{\mathrm{tP} 1}$ with a sensor in the mid-myocardial position. The modeling data also show this effect, through agreement with measurements from the sensors in the mid and distal positions, but not those closest to the surface.

Currently available catheters are not able to provide any information regarding characteristics of the deeper myocardium. The most preferred situation would be one that involves real-time monitoring of the mid-myocardial temperature profile to provide feedback on time in the resistive heating phase and thus, improved lesion formation. Further, current delivery strategies could be titrated according to the measured temperature profile to ensure operation in the linear (resistive) phase for as long as possible.

Although several strategies have been used to assess the thermal distributions during RF ablation on bench-top studies, ${ }^{[12]}$ the small dimensions and compliant mechanical properties of the ultrathin needle-type sensors reported here offer unique opportunities for use in vivo without restricting the contraction of the heart or altering the natural thermal distributions. Additionally, this platform can accommodate a variety of additional sensors to further characterize the dynamic changes of mid-myocardial tissue during ablation as valuable feedback to the clinicians.

\section{Conclusions}

While catheter ablation procedures have rapidly improved over the past several decades, limitations remain. Difficulties in real-time assessment of lesion formation frustrate optimization of ablation conditions, as current technology allows for temperature monitoring only at the myocardial surface. The flexible needle-type temperature sensors described here address this need, by enabling the in situ measurement of changes in temperature and thermal transport characteristics in real-time. Implanted sensors at multiple depths in the myocardium enhance capabilities in lesion characterization and allow discrimination of transmural and nontransmural lesions. The results suggest that temperature sensing at the myocardial surface does not readily allow prediction of lesion transmurality, likely due to the uncertain degree of convective cooling leading to inaccurate characterization of myocardial temperature profiles when compared to sensors implanted more deeply. Future 
work might allow the types of technologies reported here to be incorporated with currently available force-sensing catheters such that the ultrathin needles can be deployed into the myocardium to measure temperatures at multiple depths, simultaneously measuring the force of the catheter on the myocardium. This configuration would yield feedback data with minimal spatial artifact, during surgery. Using data from both classes of sensors, together in real-time, could allow accurate prediction of lesion transmurality and thus minimize complications.

\section{Experimental Section}

\section{Fabrication and Characterization of Ultrathin Flexible Needle-Type Temperature Sensors}

Fabrication relied on conventional microfabrication techniques. ${ }^{[7,8]}$ A polydimethylsiloxane (PDMS; $3000 \mathrm{rpm}$ for $30 \mathrm{~s}$ following with curing at $110{ }^{\circ} \mathrm{C}$ for $12 \mathrm{~min}$ ) coated glass slide served as a temporary substrate for the processing. Laminating a film of polyethylene terephthalate (PET) onto this substrate followed by photolithographic patterning and wet etching of $\mathrm{Cr}(5 \mathrm{~nm})$ and $\mathrm{Au}(150 \mathrm{~nm})$ deposited by electron beam vapour deposition in order to define the conductive traces. Photolithography and reactive ion etching (RIE) defined patterns on the PET needle. Spin-casting and curing a layer of a photocurable epoxy $(\mathrm{SU}-8,5 \mu \mathrm{m})$ formed a uniform encapsulation layer. An anisotropic conductive film (ACF) served as a cable for electrical connection to external data acquisition systems. A coating of PDMS at the interface between the film and the contacting pads on the device prevented current leakage into the surrounding biological fluids. Laser cutting of tungsten foils yielded guide tungsten needles in geometries closely matched to the devices. Cellulose served as an adhesive layer to bond these two elements together. The fabricated sensors were individually calibrated in the circulating water bath (Fisher Scientific; Waltham, MA) by comparing their resistances to readings from a standard thermometer (Hanna instrument; Woonsocket, RI). Micro X-ray computed tomography (Micro-XCT 400, Xradia, CA) yielded 3D images of the actuators/sensors. Imaging used an X-ray source at $40 \mathrm{keV}$ under $20 \times$ magnification, with 901 projections collected while rotating stepwise over $360^{\circ}$ with $8 \mathrm{~s}$ exposure time (unit voxel $=1 \mu \mathrm{m}$ ). Digital multimeters (National instruments; Austin, TX) and four-wire resistance measurement modes allowed accurate determination of the resistances of the sensors. Mechanical properties were assessed with a DMA 800 (TA instruments; New Castle, DE) using a dual/single cantilever for a rectangular film consisting identical structure of fabricated sensor, under multifrequency mode with a controlled stress (i.e., $50 \mathrm{MPa}$ ) at $37^{\circ} \mathrm{C}$.

\section{Cardiac Ablation Experiments with Perfused Rabbit Heart}

The protocol was approved by the Institutional Animal Care and Use Committee at Washington University. Experiments were performed ex vivo on hearts obtained from New Zealand White rabbits $(n=7)$. The experimental procedure has been described previously in detail. ${ }^{[13]}$ Briefly, rabbits were anesthetized intravenously with $80 \mathrm{mg} \mathrm{kg}^{-1}$ pentobarbital sodium and $400 \mathrm{U} \mathrm{kg}^{-1}$ heparin. The heart was removed and placed onto a Langendorff apparatus, where it was coronary perfused at $20 \mathrm{~mL} \mathrm{~min}^{-1}$ with oxygenated Tyrode's solution. After removing the heart from the pericardium and dissecting away epicardial fat, the heart was removed from the Langendorff apparatus and placed in a temperature- 
controlled bath $\left(37^{\circ} \mathrm{C}\right)$ with Tyrode's solution and again continuously retrograde perfused through the aorta. Distal left coronary branches were ligated and the LV free wall was dissected free by opening from apex to base along the ventricular septum. The LV free wall flap was then pinned epicardial side down onto the silicone floor of the bath. The ultrathin temperature sensors were implanted into the endocardium. Once the sensors were implanted, ablation was carried out in a circular fashion around the temperature sensor, taking care to not allow the lesions to overlap or directly contact the sensor. For radiofrequency ablation, a commercially available, nonirrigated $4 \mathrm{~mm}$ tip Safire catheter (St Jude Medical, Saint Paul, $\mathrm{MN}$ ) was used for $60 \mathrm{~s}$ at varying powers $(3-20 \mathrm{~W})$, temperature limited at $60^{\circ} \mathrm{C}$. Cryoablation was performed using an AtriCure system (AtriCure, West Chester, $\mathrm{OH}$ ), for $120 \mathrm{~s}$ at a target temperature of $-50{ }^{\circ} \mathrm{C}$. Although the heart was continuously perfused with Tyrode's solution, the concentration in the bath was adjusted to meet the resistance limitations of the RF generator. Resistance measurements of sensors were continuously measured during ablation and allowed to return to baseline between ablations. Once all endocardial surface area was exhausted, the heart was removed from the perfusion chamber and incubated in a $1 \%$ solution of triphenyltetrazolium chloride (TTC) for $20 \mathrm{~min}$ at $37^{\circ} \mathrm{C}$. The heart was then removed and lesions were transected and photographed. ${ }^{[14]}$ TTCnegative regions were classified as being successfully ablated while regions staining positive for TTC were considered viable. These images were converted to grayscale and analyzed using custom Matlab code (Mathworks, Natick, MA) to assess depth of ablation and percent transmurality of each lesion.

\section{Thermal Analysis during Cryoablation and RF Ablation}

Calibration curves allowed temperature determination from measured changes in resistances during cardiac ablation near the injection site. LabVIEW software enabled simultaneous recording at a $4 \mathrm{~Hz}$ sampling rate. ${ }^{[8]}$ Values of the thermal conductivity and heat capacity followed from analysis of transient changes in temperature associated with passage of a current of $10 \mathrm{~mA}$ for $2 \mathrm{~s}$ through the sensors using a DC current source (6620, Keithley, Cleveland, $\mathrm{OH}$ ), following details published previously. ${ }^{\left[{ }^{[9}\right.}$ Such measurements were performed before and after cardiac ablation.

\section{Temperature Profile Evaluation}

The changes in temperature associated with RF ablation were analyzed with custom Matlab codes. The first inflection point of the experimentally monitored temperature profile identifies the transition from resistive heating to conductive heating (Figure 5E). A numerical computing method for noisy data defined the second derivative of the signal. ${ }^{[15]}$ Both the timing and the temperature at this inflection point were recorded, as well as the temperature and timing of the maximum change in temperature. Two additional parameters served to characterize the process: the ratio of the time before the inflection point to the time of the maximum temperature and the ratio of the temperature increase before the inflection point to the maximum temperature. These values were statistically compared across groups and sensor depth using two-way unbalanced analysis of variance (ANOVA) method.

Statistical significance was defined as $p=0.05$. 


\section{Supplementary Material}

Refer to Web version on PubMed Central for supplementary material.

\section{Acknowledgments}

This work was supported by the NIH Grant Nos. R01 HL115415, R01 HL114395, and R21 HL112278, and through the Frederick Seitz Materials Research Laboratory at the University of Illinois at Urbana-Champaign.

\section{References}

1. Calkins H, Kuck KH, Cappato R, Brugada J, Camm AJ, Chen S-A, Crijns HJG, Damiano RJ Jr, Davies DW, DiMarco J, Edgerton J, Ellenbogen K, Ezekowitz MD, Haines DE, Haissaguerre M, Hindricks G, Iesaka Y, Jackman W, Jalife J, Jais P, Kalman J, Keane D, Kim Y-H, Kirchhof P, Klein G, Kottkamp H, Kumagai K, Lindsay BD, Mansour M, Marchlinski FE, McCarthy PM, Mont JL, Morady F, Nademanee K, Nakagawa H, Natale A, Nattel S, Packer DL, Pappone C, Prystowsky E, Raviele A, Reddy V, Ruskin JN, Shemin RJ, Tsao H-M, Wilber D. Heart Rhythm. 2012; 9:632. [PubMed: 22386883] Ceresnak SR, Perera JL, Motonaga KS, Avasarala K, Malloy-Walton L, Hanisch D, Punn R, Maeda K, Reddy VM, Doan LN, Kirby K, Dubin AM. Heart Rhythm. 2015; 12:111. [PubMed: 25277988]

2. Kowalski M, Grimes MM, Perez FJ, Kenigsberg DN, Koneru J, Kasirajan V, Wood MA, Ellenbogen KA. J. Am. Coll. Cardiol. 2012; 59:930. [PubMed: 22381429]

3. Natale A, Reddy VY, Monir G, Wilber DJ, Lindsay BD, McElderry HT, Kantipudi C, Mansour MC, Melby DP, Packer DL, Nakagawa H, Zhang B, Stagg RB, Boo LM, Marchlinski FE. J. Am. Coll. Cardiol. 2014; 64:647. [PubMed: 25125294] Reddy VY, Shah D, Kautzner J, Schmidt B, Saoudi N, Herrera C, Jaïs P, Hindricks G, Peichl P, Yulzari A, Lambert H, Neuzil P, Natale A, Kuck K-H. Heart Rhythm. 2012; 9:1789. [PubMed: 22820056]

4. Xu L, Gutbrod SR, Bonifas AP, Su Y, Sulkin MS, Lu N, Chung H-J, Jang K-I, Liu Z, Ying M, Lu C, Webb RC, Kim J-S, Laughner JI, Cheng H, Liu Y, Ameen A, Jeong J-W, Kim G-T, Huang Y, Efimov IR, Rogers JA. Nat. Commun. 2014; 5:3329. [PubMed: 24569383]

5. Kim D-H, Lu N, Ghaffari R, Kim Y-S, Lee SP, Xu L, Wu J, Kim R-H, Song J, Liu Z, Viventi J, de Graff B, Elolampi B, Mansour M, Slepian MJ, Hwang S, Moss JD, Won S-M, Huang Y, Litt B, Rogers JA. Nat. Mater. 2011; 10:316. [PubMed: 21378969]

6. Kim D-H, Ghaffari R, Lu N, Wang S, Lee SP, Keum H, D’Angelo R, Klinker L, Su Y, Lu C, Kim YS, Ameen A, Li Y, Zhang Y, de Graff B, Hsu Y-Y, Liu Z, Ruskin J, Xu L, Lu C, Omenetto FG, Huang Y, Mansour M, Slepian MJ, Rogers JA. Proc. Natl. Acad. Sci. USA. 2012; 109:19910. [PubMed: 23150574]

7. Kim T-I, McCall JG, Jung YH, Huang X, Siuda ER, Li Y, Song J, Song YM, Pao HA, Kim R-H, Lu C, Lee SD, Song I-S, Shin G, Al-Hasani R, Kim S, Tan MP, Huang Y, Omenetto FG, Rogers JA, Bruchas MR. Science. 2013; 340:211. [PubMed: 23580530]

8. Webb RC, Bonifas AP, Behnaz A, Zhang Y, Yu KJ, Cheng H, Shi M, Bian Z, Liu Z, Kim Y-S, Yeo W-H, Park JS, Song J, Li Y, Huang Y, Gorbach AM, Rogers JA. Nat. Mater. 2013; 12:938. [PubMed: 24037122]

9. Webb RC, Pielak RM, Bastien P, Ayers J, Niittynen J, Kurniawan J, Manco M, Lin A, Cho NH, Malyrchuk V, Balooch G, Rogers JA. PLoS ONE. 2015; 10:e0118131. [PubMed: 25658947]

10. Nath S, DiMarco JP, Haines DE. J. Cardiovasc. Electrophysiol. 1994; 5:863. [PubMed: 7874332]

11. Zhang H, Cheng S, He L, Zhang A, Zheng Y, Gao D. Cell Preserv. Technol. 2002; 1:141.

12. Cao H, Vorperian VR, Jang-Zern T, Tungjitkusolmun S, Je WE, Webster JG. IEEE Trans. Biomed. Eng. 2000; 47:1518. [PubMed: 11077746]

13. Nikolski V, Efimov IR. Circ. Res. 2001; 88:e23. [PubMed: 11179207]

14. Fishbein MC, Meerbaum S, Rit J, Lando U, Kanmatsuse K, Mercier JC, Corday E, Ganz W. Am. Heart J. 1981; 101:593. [PubMed: 6164281]

15. Holoborodko P. 2008 Aug. http://www.holoborodko.com/pavel/numerical-methods/numericalderivative/smooth-low-noise-differentiators/. 

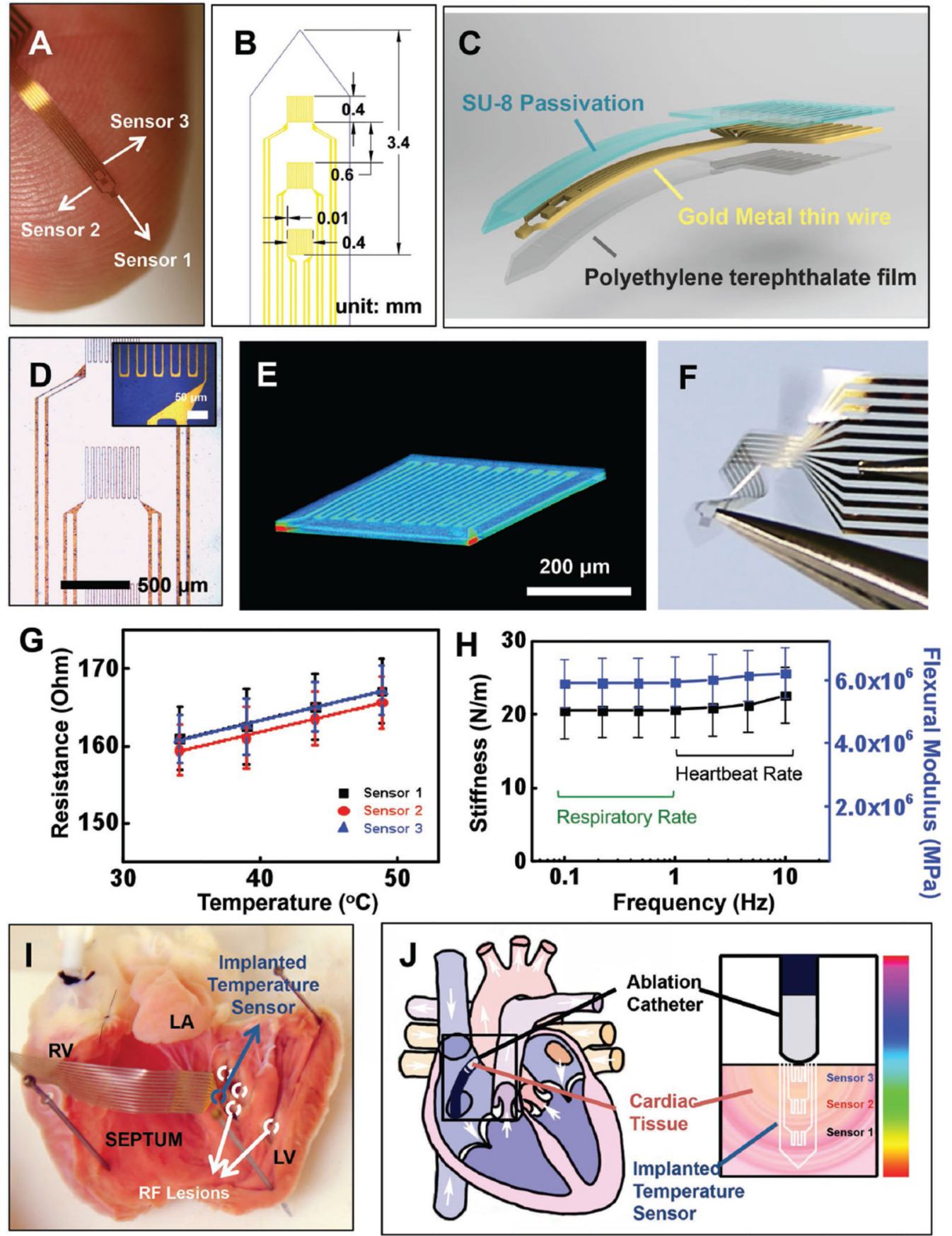

Figure 1.

Ultrathin injectable, multimodal thermal sensors for monitoring cardiac ablation in the transmural direction. A) The devices consist of three microfabricated thermistors distributed along the length of a flexible, needle-shaped platform; B) schematic illustration with key dimensions indicated; C) schematic exploded structural view of each layer of this system; D) optical images of thermal actuators/sensors on a ultrathin film of polyethylene terephthalate. Inset provides a magnified view of the edge region of a single actuator/sensor; E) X-ray tomographic images of an actuator/sensor; F) picture of completed system in a twisted 
configuration; G) calibration curve for three temperature sensors. The error bars correspond to the standard error of mean $(n=28) ; \mathrm{H})$ bending stiffness and flexural modulus of a representative device determined over a physiologically relevant range of frequencies, at $37^{\circ} \mathrm{C}$. The error bars correspond to the standard error of the mean $(n=9)$; I) image of a device injected into myocardial tissue (highlighted with blue arrow) with RF lesions (highlighted with white arrows); and J) conceptual illustration for experimental determination of transverse chronothermal profiles. 
A

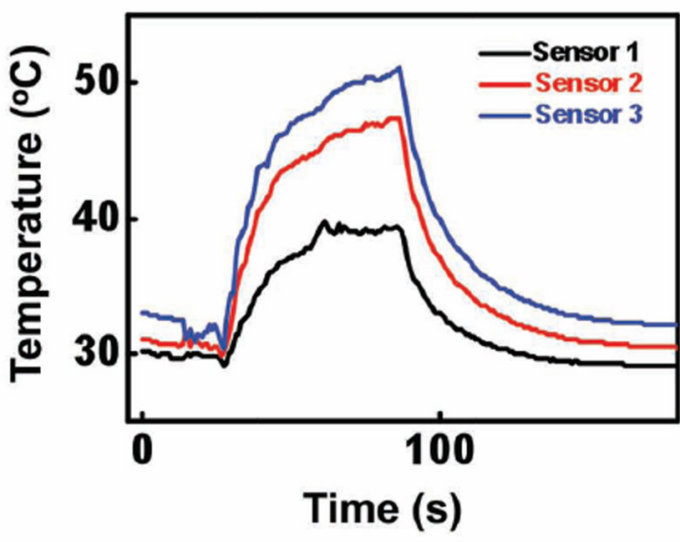

C

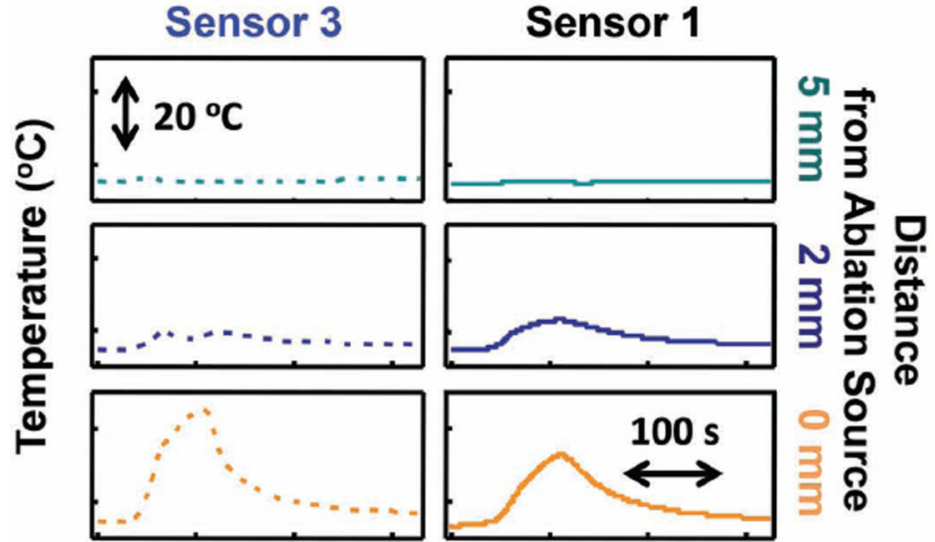

Time (s)

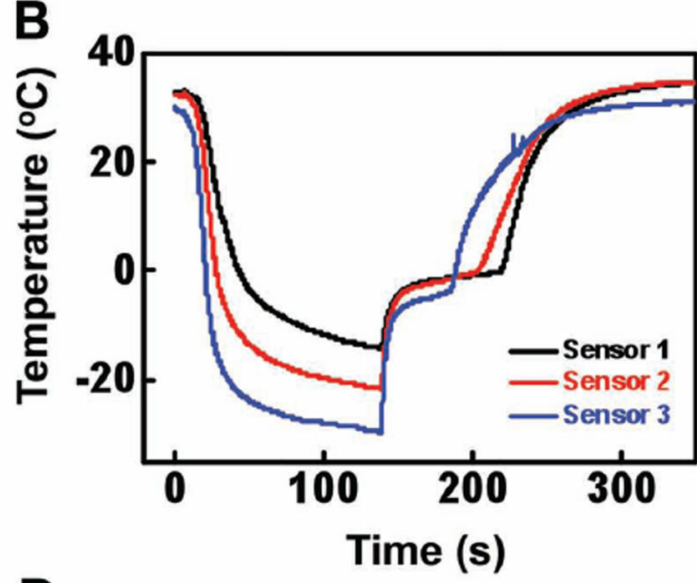

Thermal conductivity (k) Heat capacity $\left(\rho \mathrm{C}_{\mathrm{p}}\right)$ Before ablation Before ablation $\square$ After ablation After ablation

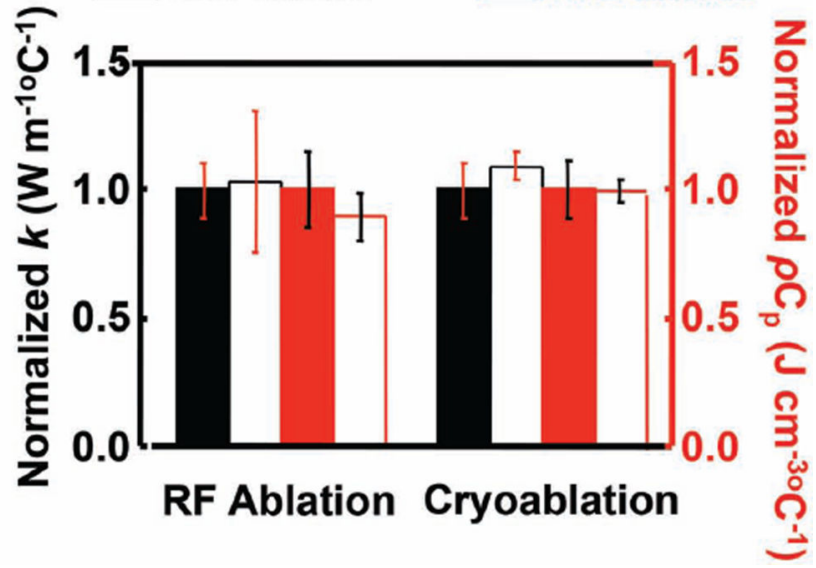

Figure 2.

Thermographic monitoring during both $\mathrm{A}$ ) radiofrequency (RF) ablation (10 W) and B) cryoablation using implanted ultrathin, flexible arrays of thermal sensors. The sensor closest to the tissue surface and ablation source corresponds to sensor 3 (blue solid line) and the one furthest from the source corresponds to sensor 1 (black solid line), per the notation in Figure 1J. C) Lateral and transverse thermographs from three temperature sensors implanted at 0 (orange), 2 (dark blue), and 5 (dark cyan) mm from the ablation source. The left column corresponds to the sensor located closest to the tissue surface (i.e., sensor 3). Results in the right column represent chronothermography of the most deeply implanted sensors (i.e., sensor 1$)$. D) Normalized thermal conductivity ( $k$; black) and volumetric heat capacity $\left(\rho C_{\mathrm{p}}\right.$; red) of cardiac tissue before and after ablation sensor. 


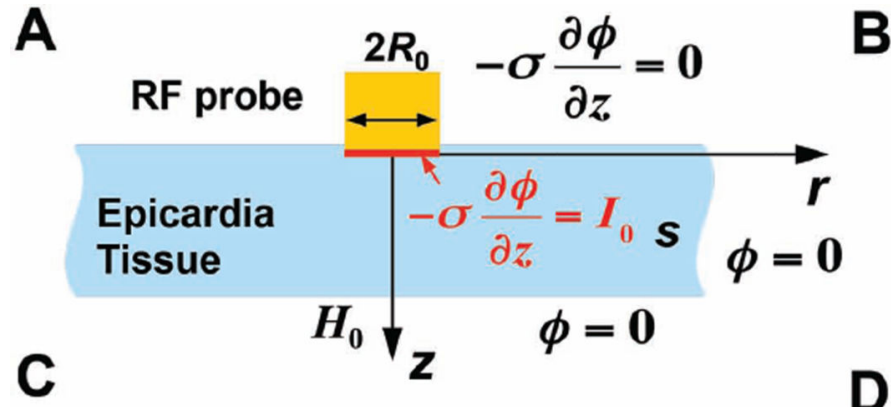

B

$$
-k \frac{\partial T}{\partial z}=h_{\mathrm{c}}\left(T-T_{\infty}\right)
$$
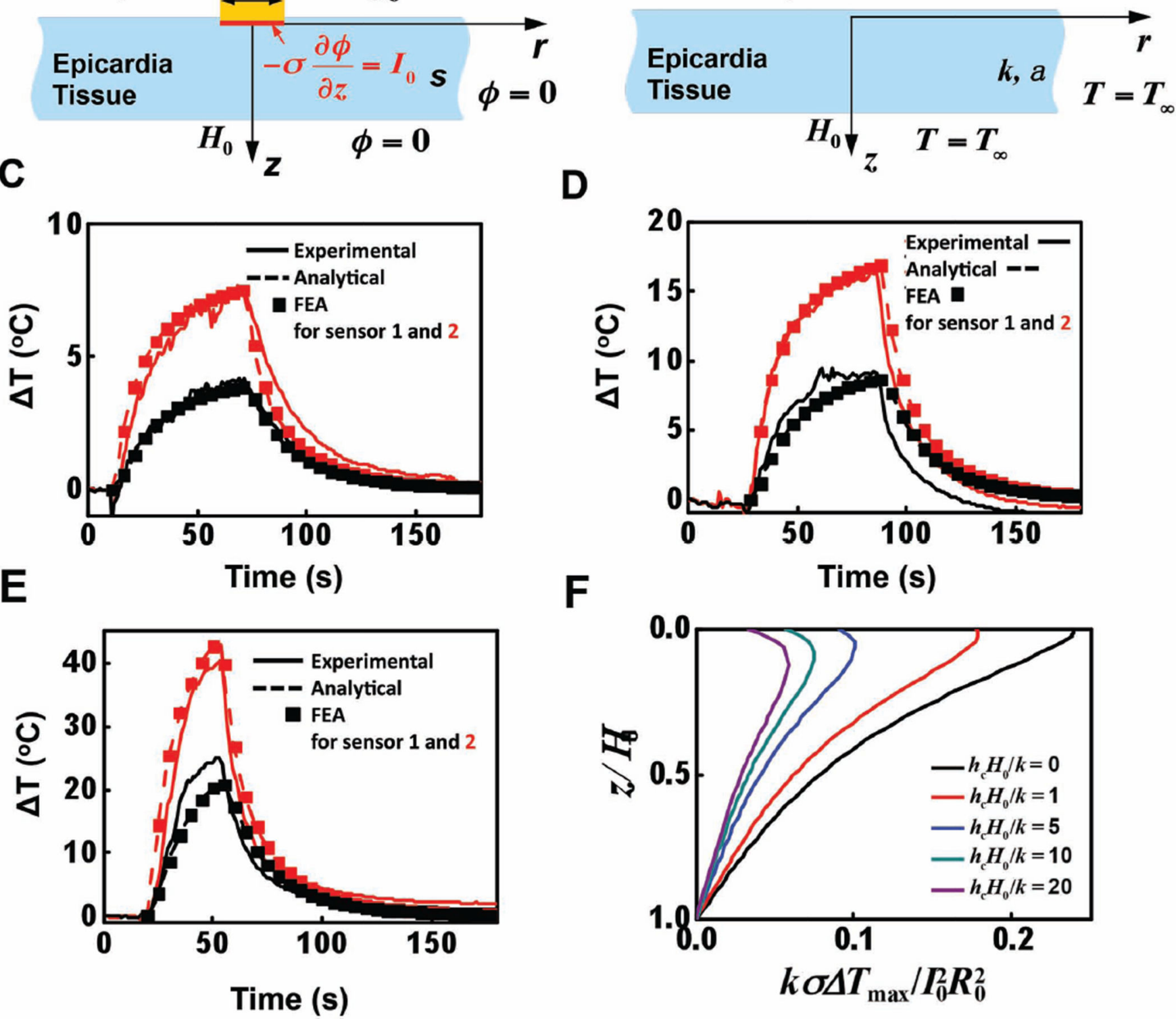

Figure 3.

Temperature change during RF ablation and computational analysis. Sketch of theoretical models for A) the electric field and B) the temperature field. Comparisons of analytical predictions (dashed line), finite element simulations (FEA; dotted), and experimental measurements (solid line) for RF ablation at C) 5, D) 10, and E) $20 \mathrm{~W}$. Computational data correspond to the predicted response of temperature sensors at the middle (sensor 2; red) and the most deeply implanted locations (sensor 1; black). F) Through-depth distributions of the normalized changes in temperature $\Delta \bar{T}=\frac{k \sigma \Delta T_{\max }(z)}{I_{0}^{2} R_{0}^{2}}$ at $\frac{r}{R_{0}}=1$ and $\frac{\alpha t_{0}}{H_{0}^{2}}=0.5$ for various relative thermal conductivity $h_{\mathrm{c}} H_{0} / k$ with $\frac{H_{0}}{R_{0}}=4$. 

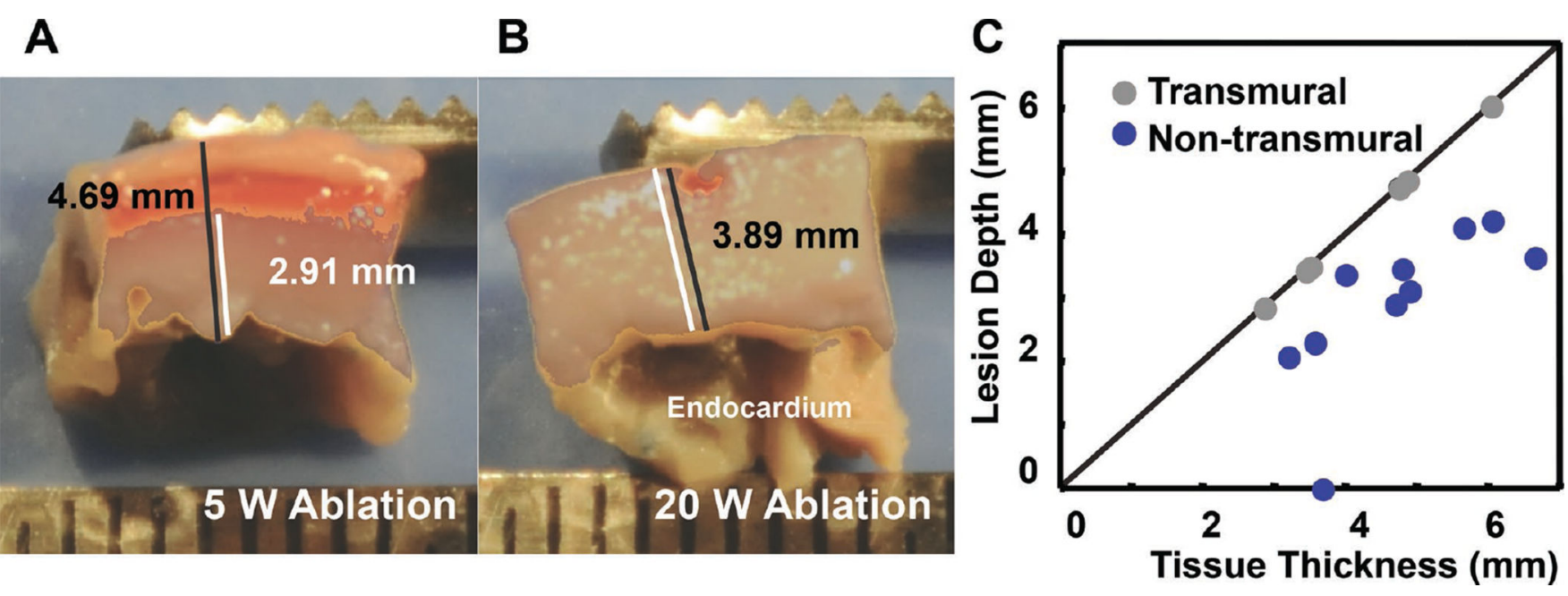

Figure 4.

Variation in lesion transmurality. Representative examples of TTC-stained lesions classified as A) nontransmural or B) transmural by custom algorithms. C) Distribution of transmurality for $n=20$ lesions included in this study. 
A
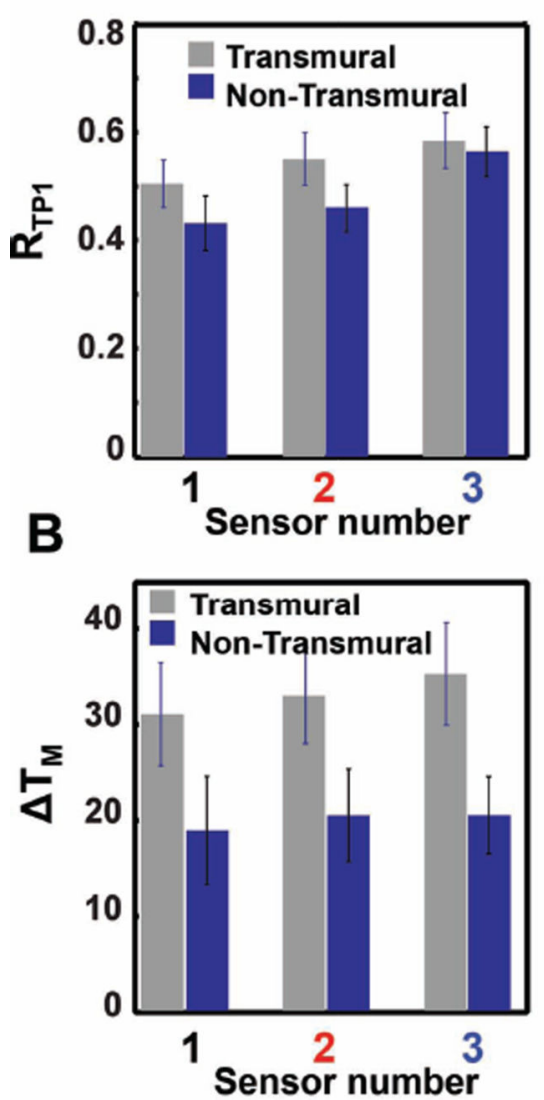

Figure 5.
C
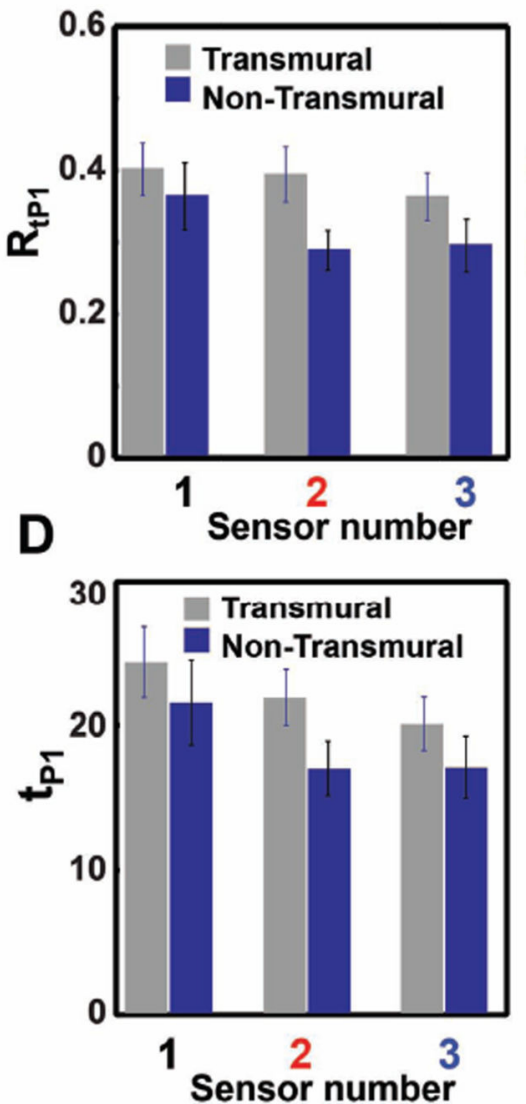

E
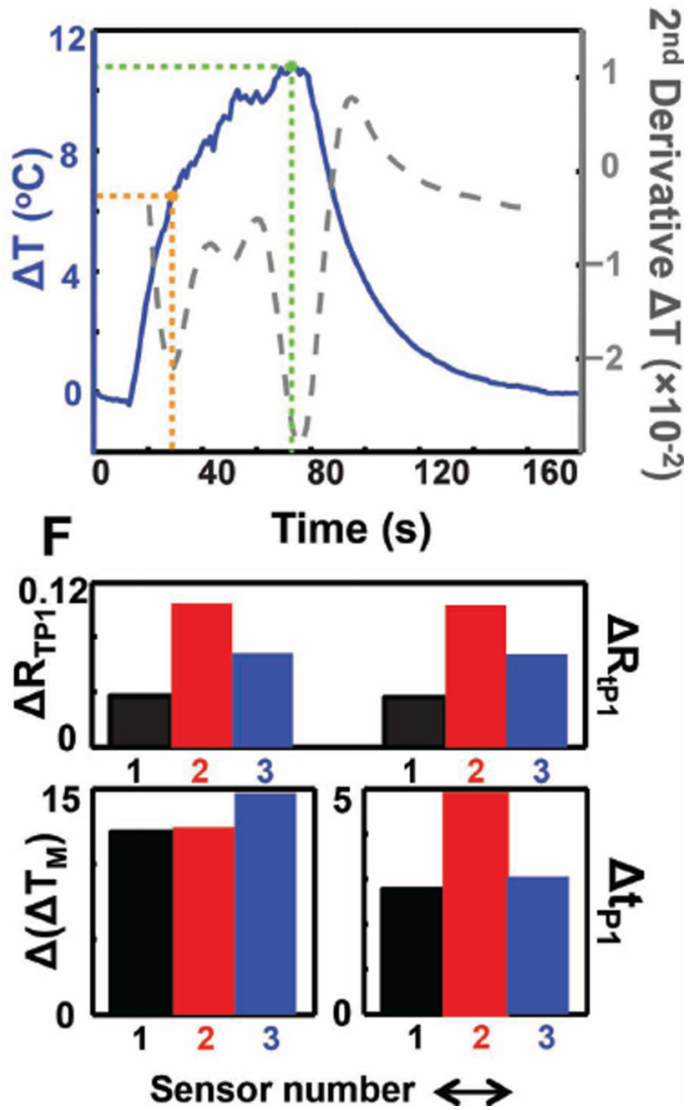

Comparison of chronothermograph parameters for nontransmural and transmural lesions and across sensor locations assessed by A) the ratio of the temperature change in phase 1 to the maximum temperature change $\left(R_{\mathrm{TP} 1}\right)$; B) the maximum change in temperature $\left.\left(\Delta T_{\mathrm{M}}\right) ; \mathrm{C}\right)$ the ratio of the time duration in phase 1 to time maximum temperature was achieved $\left(R_{\mathrm{tP} 1}\right)$; and D) the time spent in phase $1\left(t_{\mathrm{P} 1}\right)$. E) Representative chronothermograph (blue) and second derivative (gray) from which parameters were extracted. Orange identifies the inflection point (phase 1) and green identifies the maximum temperature. F) Differences across the transmural group for each parameter and sensor location. 

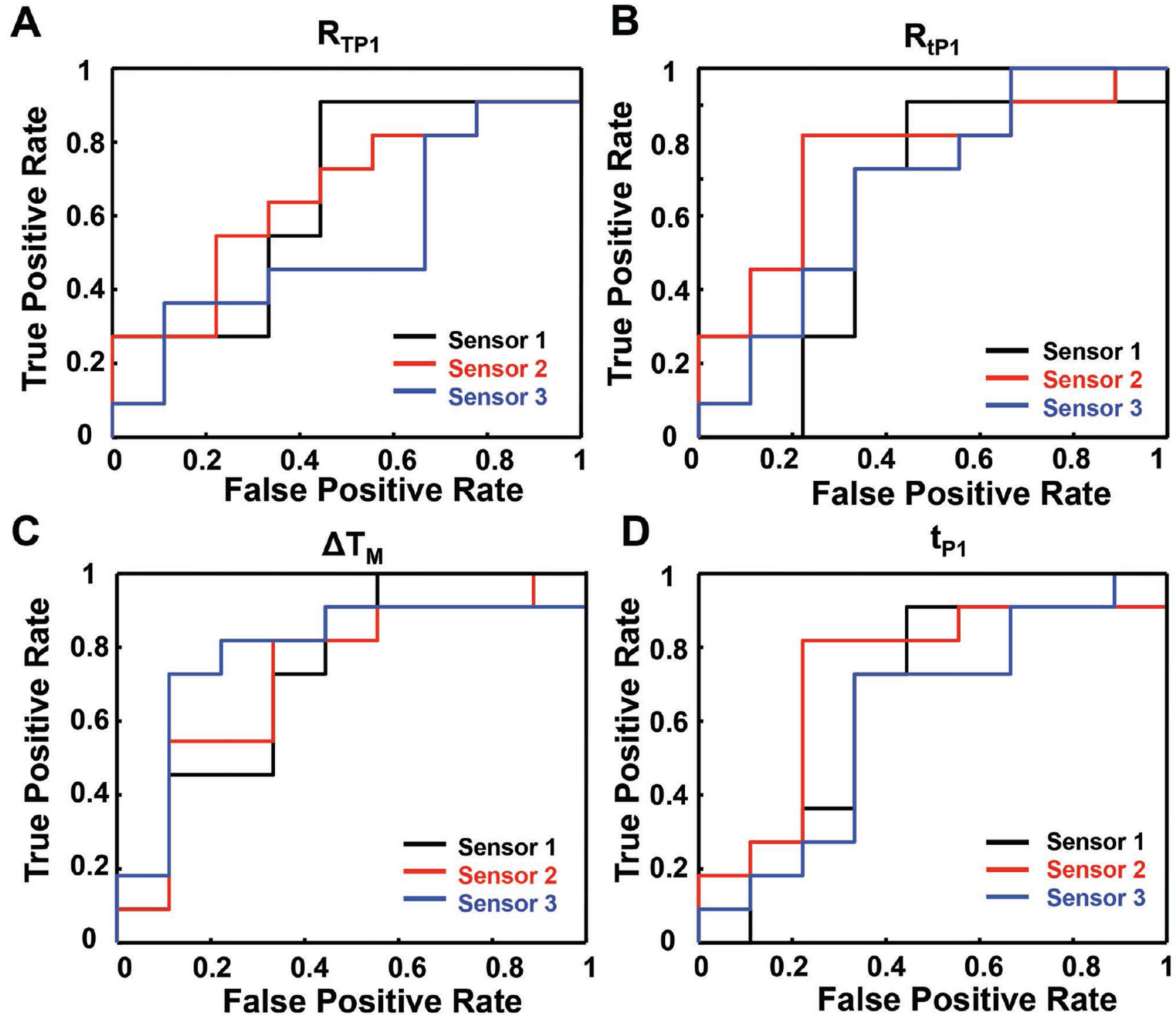

Figure 6.

Predictive parameters for transmural lesions. Receiver operating characteristic (ROC) curves across all sensor locations for: A) the ratio of temperature change in phase 1 to maximum temperature change $\left.\left(R_{\mathrm{TP} 1}\right) ; \mathrm{B}\right)$ the ratio of time duration in phase 1 to time maximum temperature was achieved $\left.\left(R_{\mathrm{tP} 1}\right) ; \mathrm{C}\right)$ the maximum temperature $\left(\Delta T_{\mathrm{M}}\right)$; and $\left.\mathrm{D}\right)$ the time spent in phase $1\left(t_{\mathrm{P} 1}\right)$. 


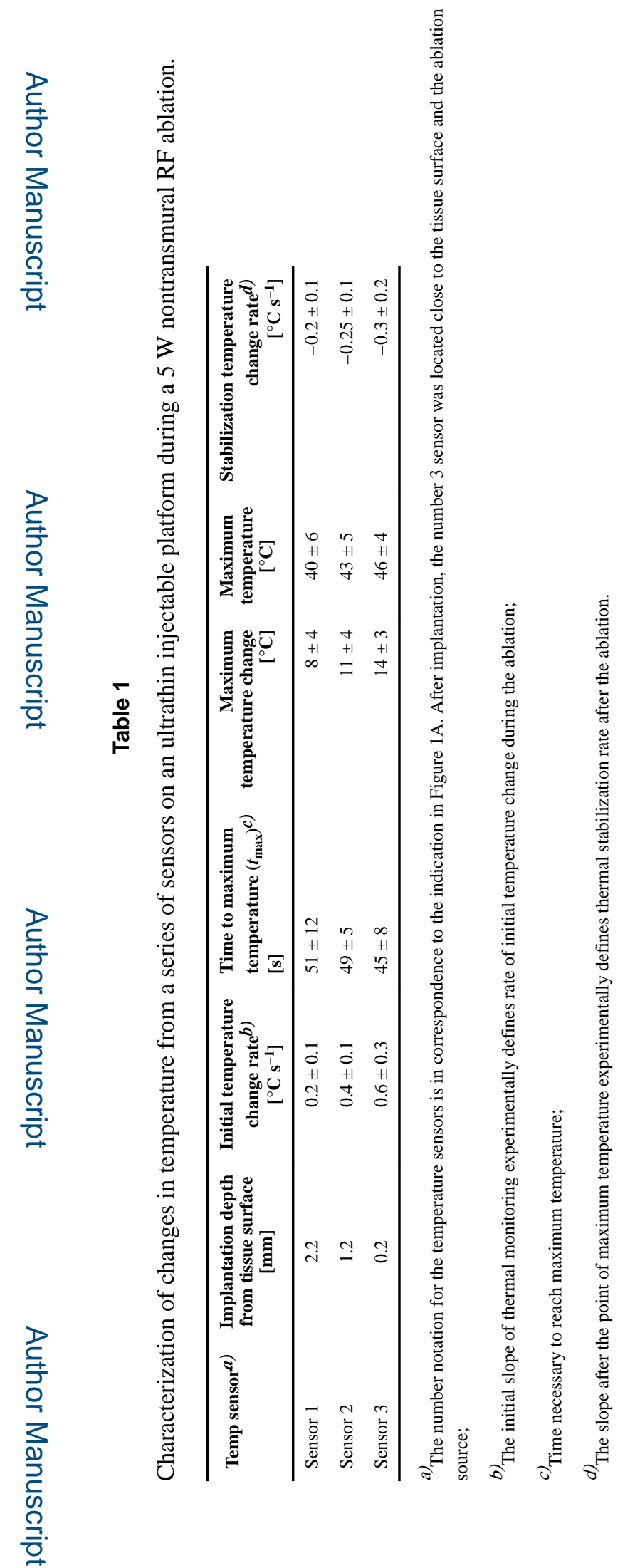

Adv Healthc Mater. Author manuscript; available in PMC 2017 February 04. 


\section{Table 2}

Areas under the curve (AUCs) for the receiver operation characteristic (ROC) curves presented in Figure 6.

\begin{tabular}{lccc}
\hline & Sensor 1 & Sensor 2 & Sensor 3 \\
\hline Ratio of temperature at phase 1 & 0.66 & 0.66 & 0.53 \\
Ratio of time to phase 1 & 0.62 & 0.76 & 0.68 \\
Maximum temperature change & 0.74 & 0.73 & 0.79 \\
Time to phase 1 & 0.66 & 0.73 & 0.62 \\
\hline
\end{tabular}

